\title{
Papers
}

\section{Autopsy after termination of pregnancy for fetal anomaly: retrospective cohort study}

\author{
P A Boyd, F Tondi, N R Hicks, P F Chamberlain
}

\begin{abstract}
Objective To study trends in termination of pregnancy for fetal anomaly over 10 years and to assess the contribution of autopsy to the final diagnosis and counselling after termination.

Design Retrospective study with cases from a congenital anomaly register and a defined unselected population. Data sources Pregnancies resulting in termination for fetal anomaly identified from the Oxford congenital anomaly register. Details about the prenatal diagnosis and autopsy findings were retrieved from case notes.

Results Of the 57258 deliveries, $309(0.5 \%)$ were terminated because of prenatally diagnosed abnormality. There were $129 / 29086(0.4 \%)$ terminations for fetal anomaly carried out in 1991-5 and 180/28 $172(0.6 \%)$ in 1996-2000. The percentage of fetuses that underwent autopsy fell from $84 \%$ to $67 \%$. Autopsy was performed in 132 cases identified by ultrasound scan, with no evidence for abnormal karyotype. In $95(72 \%)$ the autopsy confirmed the suspected diagnosis and did not add important further information, two cases were not classified, and in 35 (27\%) the autopsy added information that led to a refinement of the risk of recurrence (reduced in 17, increased in 18); in 11 of these 18 cases it was increased to a one in four risk.

Conclusions Though there has been an increase in the rate of terminations of pregnancy for fetal anomaly, there has been a decline in the autopsy rate. When a prenatal diagnosis was based on the results of a scan only, the addition of information from an autopsy by a specialist paediatric pathologist provided important information that changed the estimated risk of recurrence in $27 \%$ of cases and in $8 \%$ this was to a higher (one in four) risk.
\end{abstract}

\section{Introduction}

The Abortion Act 1967 allows termination of pregnancy if there is substantial risk that if the child were born it would suffer from such physical or mental abnormalities as to be seriously disabled. During the past decade new screening and diagnostic tests have been introduced that allow earlier and more accurate diagnosis of fetal anomaly. ${ }^{1-3}$

When a serious anomaly is suspected prenatally some parents request termination of pregnancy. This request may be based on the results of investigations that imply that the baby will almost certainly have a lethal anomaly-for example, anencephaly-or one likely to cause long term morbidity-for example, spina bifida. In other instances termination of pregnancy may be requested when the implications of the investigations are less clear-for example, a fetus with mild cerebral ventriculomegaly. After termination of pregnancy most parents will want to know if the prenatal prediction was accurate and what the implications are for future pregnancies. This is particularly true when the prenatal diagnosis is based on ultrasound scan findings only. Such information may be obtained from the autopsy examination.

After the adverse publicity surrounding paediatric autopsies at Alder Hey Hospital ${ }^{4}$ there is widespread recognition that parents need a full explanation to make an informed decision. This information should not only be about the autopsy technique but also about the potential benefits. Currently there is little quantitative information about the likelihood of autopsy being of practical use to parents and their families by modifying the assessment of the risk of recurrence.

The prenatal diagnosis unit in Oxford is a tertiary referral centre in a teaching hospital. Since 1991 all anomalies (with the exception of those on the Office for National Statistics exclusion list $\mathrm{t}^{5}$ ) suspected prenatally and those presenting postnatally have been recorded on the Oxford congenital anomaly register (OXCAR) with the outcome of each pregnancy. We used 10 years of data reported to the register ${ }^{6} 7$ to assess how often information from the autopsy changes the suspected prenatal diagnosis and hence the advice given to parents about risks of recurrence; to ascertain the rates of termination of pregnancy and autopsy over 10 years in cases of prenatally suspected anomalies; to report on the range of anomalies for which termination of pregnancy was carried out and their severity; and to estimate the reduction in prevalence of conditions associated with long term morbidity because of prenatal diagnosis and termination of pregnancy.

\section{Methods}

In the early 1990s, in common with most centres, local women were offered $\alpha$ fetoprotein screening for neural tube defects at 15-18 weeks and an anomaly scan at 18-22 weeks of gestation. This is still current policy. By the late 1990s most centres nationally offered some form of screening for Down's syndrome (other than by maternal age) on the NHS but neither nuchal scans nor first or second trimester biochemical screening for Down's syndrome are available in Oxford, although some women pay for these tests privately. Throughout the study period chorionic villus sampling or amniocentesis was offered to women aged over 35 years at the expected date of delivery. In Oxford autopsies are carried out by specialist paediatric pathologists.

From the register we identified all women with an OX postcode who were booked for delivery at the John Radcliffe Women's Centre or a local community hospital with a date of delivery between 1 January 1991 and 31 December 2000 inclu- 


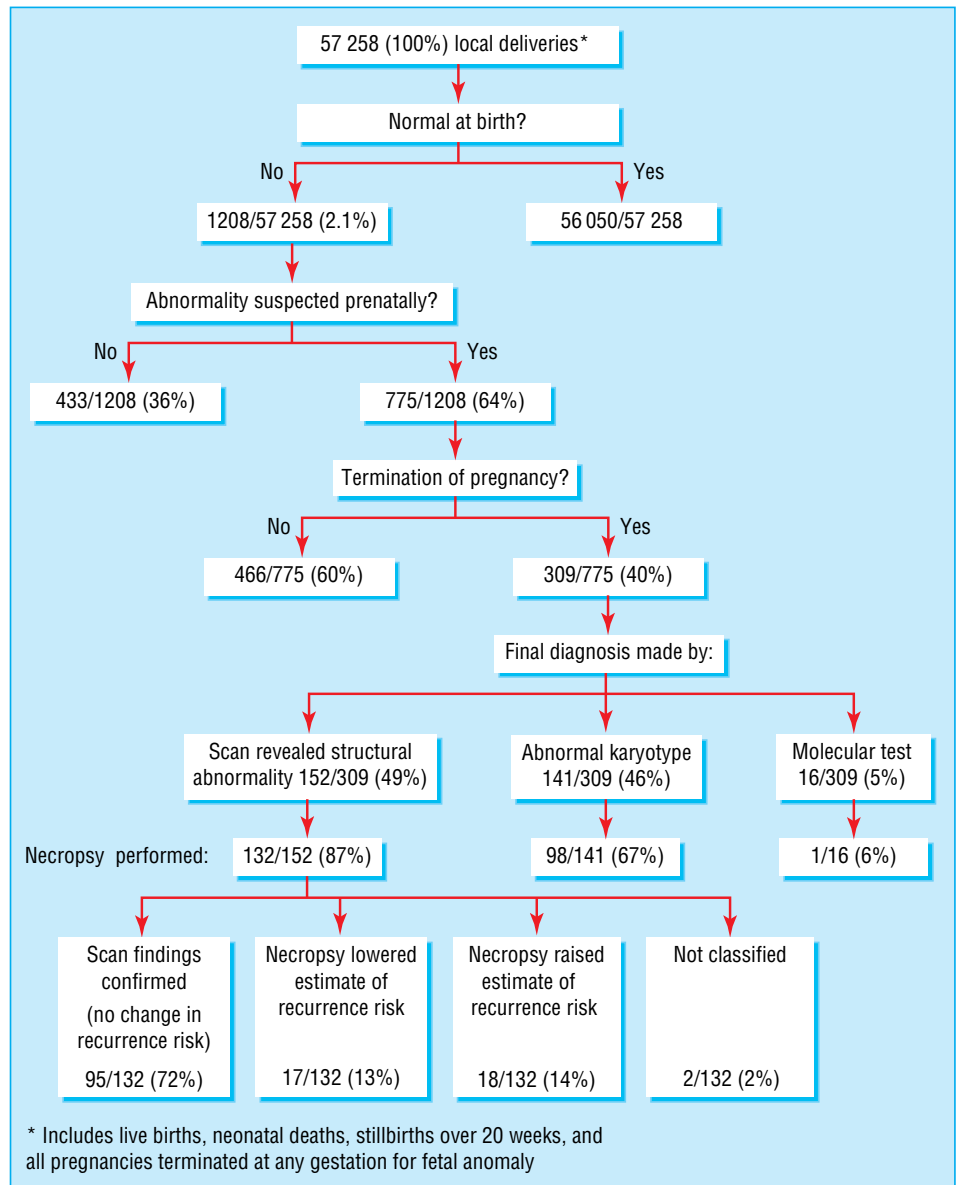

Fig 1 Summary chart of prenatal screening and termination of pregnancy for congenital abnormality in Oxford, 1991-2000, inclusive

sive and in whom the outcome of pregnancy was recorded as termination. We excluded women booked into and referred from other hospitals outside the catchment area because of test results that indicated high risk for fetal abnormality and those with an OX postcode who (had the pregnancy continued) would normally have delivered at another district hospital. The study population was therefore unselected. The total number of deliveries occurring in the same population and time period was obtained from the Oxford Maternity Data System. Completeness of ascertainment was checked by searching records in genetics laboratories and clinical genetics and histopathology units and with the maternity data system. We reviewed autopsy reports, clinical notes, and laboratory and ultrasound reports.

We identified those cases in which a structural anomaly was diagnosed by scan, there was no evidence for abnormal karyotype, and an autopsy was carried out. Two investigators (PAB, PFC) reviewed the autopsy findings to determine whether they altered the suspected prenatal diagnosis and the assessment of the risk of recurrence (see table 2 for details). We discussed discrepancies between investigators before we agreed final categorisation.

\section{Results}

Figure 1 provides an overview of the prenatal diagnosis of congenital anomalies and termination of pregnancy for fetal anomaly in local deliveries in Oxford during the 10 years 19912000 inclusive. Of the 57258 deliveries (live births, neonatal deaths, stillbirths over 20 weeks, and all pregnancies terminated at any gestation for fetal anomaly), 1208 (2.1\%) resulted in a fetus or baby with an abnormality.

Of the 57258 births, $309 \quad(0.5 \%)$ were terminated pregnancies because a fetal anomaly had been suspected or diagnosed prenatally. The rate and number of terminations for suspected fetal anomaly increased during the study period, from $129(129 / 29086(0.4 \%))$ in $1991-5$ to $180(180 / 28172(0.6 \%))$ in 1996-2000. In the same time periods the number of prenatal diagnoses made (with abnormality present at birth) increased from $41 \%$ to $46 \%{ }^{7}$. The investigation that led to the final diagnosis being made was ultrasound scan (that is, structural anomaly, no evidence for chromosome anomaly) in 152 (49\%) cases, abnormal karyotype (by chorionic villus sampling, amniocentesis, or fetal blood sampling) in $141(46 \%)$, and molecular testing of DNA in $16(5 \%)$.

Figure 2 shows that the number of terminations of pregnancy in which autopsy was carried out fell from $84 \%$ in the first five years to $67 \%$ in the second five years.

Table 1 gives the final postnatal diagnosis for those fetuses from terminated pregnancies grouped by the body system involved and by lethality as previously described. ${ }^{6}$ Chromosome anomalies accounted for 141 (46\%) of the 309 cases. Neural tube defects and other central nervous system defects were the most common structural defects, accounting for a further 66 (21\%) cases. One hundred and thirty three (43\%) fetuses were considered to have defects which would have proved lethal, and $44(14 \%)$ defects were possibly lethal. In $132(43 \%)$ the defects were compatible with survival beyond a year. 


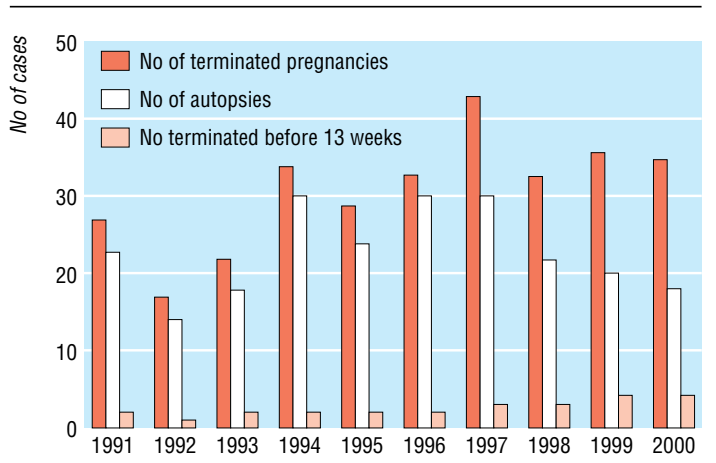

Year

Fig 2 Number of pregnancies terminated because of fetal anomaly by year $(n=309)$, number of autopsies, and number terminated before 13 weeks' gestation (autopsy not possible before 14 weeks)

In this population during the 10 years of the study there were 1208 fetuses or babies with a malformation. Of these, 601 had a malformation compatible with survival with long term morbidity. If we assume that half of those 44 fetuses with defects classified as possibly lethal (for example, diaphragmatic hernia) did survive (that is, 154 survivors in all), the reduction in prevalence of congenital malformations associated with long term morbidity due to termination of pregnancy would be $26 \%$ (that is, $154 / 601$ ). The true reduction in prevalence would be lower than this because some of the pregnancies would have ended in miscarriage (for example, 43\% of Down's syndrome cases diagnosed between the time of chorionic villus sampling and term or $23 \%$ between the time of amniocentesis and term ${ }^{8}$ ).

Table 2 shows an assessment of the contribution of the autopsy findings to the final diagnosis made for the purpose of counselling after termination of pregnancy for the 132 cases with structural anomalies and no evidence for abnormal karyotype. Autopsy findings led to a refinement of the risk of recurrence (category 3) in $35(27 \%)$ cases. In 11/18 (61\%) (category 3b) of those in which the autopsy findings led to an increase in the estimated risk of recurrence this was to a probable one in four risk for subsequent pregnancies. Two of the 132 cases (category 4) could not be classified; termination of pregnancy had been carried out at the parents' request before test results (subsequently normal) had been reported as described previously. ${ }^{6}$

\section{Discussion}

\section{Autopsy rates}

In an unselected population over a 10 year period there has been a decline in the number of fetal autopsies carried out (16\% had no autopsy in the first five years and 34\% in the second) despite an increase in the number of terminations of pregnancy after prenatal suspicion of fetal abnormality. Though this decline preceded the adverse publicity surrounding events at Alder Hey Hospital, it has accelerated since. ${ }^{4} \mathrm{~A}$ fall in autopsy rates has also been reported by others.

Contribution of the autopsy to estimation of recurrence risks When parents make a difficult decision to terminate a pregnancy because of suspected fetal abnormality most want to know if the prenatal suspicions are verified and what the implications are for further pregnancies for themselves and their wider families. We have shown that when the final prenatal diagnosis was made by ultrasound scan, in $27 \%$ of cases the information from the autopsy examination led to a refinement of the risk of recurrence, and in $8 \%$ this was increased to a one in four risk. We believe that these data may be of particular value to parents, and to those counselling them, when they are faced with a decision about whether to proceed with fetal autopsy after termination of pregnancy for suspected fetal anomaly. Our study of an unselected population took place at a tertiary referral centre with autopsy performed by specialist paediatric pathologists. Care should be taken in extrapolating these data to other centres, especially those without access to a specialist paediatric pathologist. While consistent with results of other studies, ${ }^{9}{ }^{10}$ our findings are important because they are based on a defined unselected population and provide practical information for parents concerning the chance of acquiring new information on risks in subsequent pregnancies.

In a study of neonatal autopsy Brodlie et al found a similar proportion in which new information was revealed, ${ }^{9}$ although this is not directly comparable with the current study of termination of pregnancy because of the influence of postnatal events and management. In a study of 300 fetal autopsies, Laussell-Riera et al found that the autopsy examination changed the prenatal "hypothesis" in 20\%, provided extensive additional information in $41 \%$, and confirmed the prenatal hypothesis in $39 \%{ }^{10}$

\section{Contribution of prenatal diagnosis to reduction in prevalence of non-lethal congenital anomalies}

In 1998 we reported that in about half of the cases of termination of pregnancy the fetal abnormality would have proved lethal and in half the abnormality was compatible with survival beyond the first year. ${ }^{6}$ We have now added four more years of data from the same population. Recalculation of the crude reduction in prevalence of congenital anomaly associated with long term morbidity because of termination of pregnancy shows it to have increased from $20 \%$ to $26 \%$.

\section{Benefits of autopsy}

Quantifying the value of autopsy is not easy. For example, in many cases of lethal skeletal dysplasia a diagnosis can be attempted prenatally, ${ }^{11}$ but confirmation is needed from autopsy and $\mathrm{x}$ ray studies and these may change the suspected risk of recurrence from low (for example, thanatophoric dysplasia) to high (for example, Jeune's syndrome). Similarly renal cystic disease may be difficult to define on a scan because of a lack of amniotic fluid, and the differentiation between infantile polycystic kidney disease (recurrence risk 25\%) and cystic renal dysplasia (recurrence risk $3 \%$ ) may require histological examination.

The direct benefits of autopsy to parents are not limited to refining the risk of recurrence. Even after autopsy, sometimes a definitive final diagnosis cannot be made and information given to parents may cover a range of possible diagnoses. In such cases the storage of fetal samples for possible future genetic analysis provides the hope of an accurate diagnosis (which may have ramifications for the wider family) at a much later date. In most cases in which the scan findings are confirmed parents can gain comfort that their baby had the prenatally suspected condition. The finding of additional malformations, as well as in some cases changing the diagnosis, may be helpful in targeting tests in a subsequent pregnancy. A wider importance of autopsy is in its value for quality control for prenatal diagnosis, teaching, and research.

The decline in autopsy rate and issues surrounding the retention of tissues and organs for diagnostic studies, teaching, and research has been the subject of much debate since the adverse publicity concerning autopsies and organ retention. ${ }^{12-15}$ 
Table 1 Number of pregnancies terminated broadly categorised by defect and lethality

\begin{tabular}{|c|c|c|c|c|}
\hline Main defect/system & Lethal & Possibly lethal & Possible survivor & Total \\
\hline Neural tube defect/other central nervous system: & & & & $66(21 \%)$ \\
\hline Anencephaly & 26 & - & - & \\
\hline Encephalocele & 1 & - & - & \\
\hline Hydrocephalus/other & - & 3 & - & \\
\hline Spina bifida & - & - & 30 & \\
\hline Other & 1 & - & 5 & \\
\hline Chromosomes: abnormal karyotype & 53 & 8 & 80 & $141 \quad(46 \%)$ \\
\hline Cardiac: & & & & $8 \quad(3 \%)$ \\
\hline Hypoplastic left heart & 4 & - & - & \\
\hline Complex cardiac & - & 3 & - & \\
\hline Tetralogy of Fallot & - & - & 1 & \\
\hline Renal: & & & & $(3 \%)$ \\
\hline Renal agenesis/cystic dysplasia & 8 & - & - & \\
\hline $\begin{array}{l}\text { Obstructive renal } \\
\end{array}$ & - & 2 & - & \\
\hline Skeleton (includes some inherited disorders): & & & & $16 \quad(5 \%)$ \\
\hline Skeletal dysplasia & 9 & 5 & - & \\
\hline Skeletal limb reduction & - & - & 2 & \\
\hline Anterior abdominal wall/diaphragm: & & & & $(2 \%)$ \\
\hline Diaphragmatic hernia & - & 4 & - & \\
\hline Exomphalos & - & - & 1 & \\
\hline Hydrops/cystic hygroma: & & & & $\begin{array}{ll}6 & (2 \%)\end{array}$ \\
\hline Hydrops & 3 & - & - & \\
\hline Cystic hygroma & & 1 & 2 & \\
\hline Multiple abnormalities & 13 & 2 & - & $15 \quad(5 \%)$ \\
\hline Inherited disorders: & & & & $(8 \%)$ \\
\hline Meckel-Gruber syndrome & 5 & - & - & \\
\hline Multiple pterygium syndrome & 1 & - & - & \\
\hline Orofaciodigital syndrome & 1 & - & - & \\
\hline Myotonic dystrophy & - & 5 & - & \\
\hline Donohue's syndrome & - & 1 & - & \\
\hline Spinal muscular atrophy & - & 4 & - & \\
\hline Ornithine transcarbolamine deficiency & - & 1 & - & \\
\hline Unknown recessive & - & 1 & 2 & \\
\hline Sickle cell & - & - & 1 & \\
\hline Duchenne's muscular dystrophy & - & - & 1 & \\
\hline Adrenogen insensitivity syndrome & - & - & 1 & \\
\hline$\alpha_{1}$ antitrypsin deficiency & - & - & 1 & \\
\hline Fragile X & - & - & 1 & \\
\hline Other: & & & & $16 \quad(5 \%)$ \\
\hline Amniotic bands, conjoined twins, ?syndrome & 8 & - & - & \\
\hline Teratoma & - & 2 & - & \\
\hline ?syndromes & - & 2 & - & \\
\hline Normally formed fetus $^{*}$ & - & - & 2 & \\
\hline Cystadenomatous lesion of lung & - & - & 1 & \\
\hline Cornelia de Lange syndrome & - & - & 1 & \\
\hline Total & $133(43 \%)$ & $44(14 \%)$ & $132(43 \%)$ & $309(100 \%)$ \\
\hline
\end{tabular}

*See text.

Parents should be provided with full information and not be coerced into accepting an autopsy examination. It is important that those advising them at such a sensitive time do not take what may be the superficially kinder route of avoiding detailed discussion about the autopsy. Parents need full information about the potential benefits of the examination, including details both about the procedures involved and about the benefits in providing information about risks of recurrence if they are to make a truly informed decision. This discussion should be with an appropriately trained professional. Our study provides important information for parents. If a termination has been carried out because of anomalies detected by ultrasound scan, by declining an autopsy, parents will remain ignorant of information that might change the recurrence risk in one in four cases and have a one in 13 chance for missing confirmation of a high (one in four) recurrence risk.
We thank CA Boyd for advice and reading the manuscript and S Gould and C Bowker (paediatric pathologists), A Roberts (specialist genetics nurse), S Hanson (data systems manager), and all the staff in the Oxford Prenatal Diagnosis Unit for help and for providing information.

Contributors: $\mathrm{PAB}$ and PFC initiated the research. FT collected and collated the data. PAB, PFC, and NRH analysed and interpreted the data.. PAB wrote the paper with contributions from NRH and PFC. PAB is guarantor.

Funding: No additional funding.

Competing interests: None declared.

Ethical approval: Sought but not required by local committee.

1 Royal College of Obstetricians and Gynaecologists. Ultrasound screening for fetal abnormalities: report of the RCOG working party. London: RCOG, 1997.

2 Wald NJ, Kennard A, Hackshaw A, McGuire A. Antenatal screening for Down's syndrome. A review. Health Technol Assess 1998;2:1-112.

3 Bricker L, Garcia J, Henderson J, Mugford M, Neilson J, Roberts T, et al. Ultrasound screening in pregnancy: a systematic review of the clinical effectiveness, cost-effectiveness and women's views. Health Technol Assess 2000;4:1-193. 
Table 2 Contribution of autopsy in 132 cases of termination of pregnancy due to structural anomalies detected at scan with no evidence for abnormal karyotype and autopsy performed

\begin{tabular}{lccc} 
Category & Contribution of autopsy & No (\%) of cases & Example \\
\hline 1 & $\begin{array}{c}\text { Prenatal diagnosis } \\
\text { confirmed; scan and } \\
\text { autopsy findings } \\
\text { identical }\end{array}$ & $72(55 \%)$ & $\begin{array}{c}\text { Scan=isolated } \\
\text { anencephaly; autopsy } \\
\text { =isolated anencephaly }\end{array}$ \\
\hline 2 & $\begin{array}{c}\text { Prenatal diagnosis } \\
\text { confirmed; autopsy } \\
\text { added some information } \\
\text { which did not change } \\
\text { recurrence risk }\end{array}$ & $23(17 \%)$ & $\begin{array}{c}\text { Scan=anencephaly; } \\
\text { autopsy =anencephaly + } \\
\text { unilateral renal agenesis }\end{array}$ \\
\hline $3 \mathrm{a}$ & $\begin{array}{c}\text { Autopsy added } \\
\text { information that reduced } \\
\text { estimate of recurrence } \\
\text { risk }\end{array}$ & $17(13 \%)$ & $\begin{array}{c}\text { Scan=anencephaly; } \\
\text { autopsy =amniotic band }\end{array}$ \\
\hline $3 \mathrm{~b}$ & $\begin{array}{c}\text { Autopsy added } \\
\text { information that } \\
\text { increased estimate of } \\
\text { recurrence risk }\end{array}$ & $18(14 \%)$ & $\begin{array}{c}\text { Scan=cystic kidneys; } \\
\text { autopsy =infantile } \\
\text { polycystic kidney } \\
\text { disease }\end{array}$ \\
\hline 4 & Other (see text) & $2(2 \%)$ &
\end{tabular}

4 Redfern M, Keeling JW, Powell E. The Royal Liverpool Children's inquiry report. London: Stationery Office, 2001.

Congenital malformation statistical notifications 1994.London: HMSO, 1997.(Series MB3 No 10 .)

6 Boyd PA, Chamberlain P, Hicks NR. Six year experience of prenatal diagnosis in an unselected population in Oxford, UK. Lancet 1998;352:1577-81.

7 National Perinatal Epidemiology Unit. Report of the Oxford Congenital Anomaly Register 1991-2001. Oxford: National Perinatal Epidemiology Unit, 2003.

8 Morris JK, Wald NJ, Watt HC. Fetal loss in Down syndrome pregnancies. Prenat Diag 1999;19:142-5.

9 Brodlie M, Laing IA, Keeling JW, McKenzie KJ. Ten years of neonatal autopsies in tertiary referral centre: retrospective study. BMJ 2002;324:761-3.

10 Laussel-Riera A, Devisme L, Manouvrier-Hanu S, Puech F, Robert Y, Gosselin B. Value of fetopathological examination in medical abortions: comparison of prenatal diagnosis and autopsy results of 300 fetuses. Ann Pathol 2000:20:549-57.

11 Gaffney G, Manning N, Gould S, Boyd PA, Chamberlain P. An ultrasonographic assessment of skeletal dysplasias-a report of the diagnostic and prognostic accuracy in 35 ment of skeletal dysplasias - a report

12 Khong TY. Falling neonatal autopsy rates BMJ 2002;324:749-50.

13 Hunter M. Alder Hey report condemns doctors, management, and coroner. BMJ 2000;322:255.

14 Anonymous. Families need more information about postmortem examinations. $B M J$ 2002;325:1121.

\section{What is already known on this topic}

After prenatal diagnosis of fetal anomaly some parents opt for termination of pregnancy

The rate of decline in the uptake of autopsy has accelerated since adverse publicity at Alder Hey Hospital

\section{What this study adds}

The number of pregnancies resulting in termination after prenatal diagnosis of fetal anomaly has increased over a 10 year period

When termination of pregnancy occurs after identification of structural anomalies on scan and there is no evidence for abnormal karyotype, new information from autopsy changes the estimated risk for a recurrence in more than a quarter of cases; in $8 \%$ this is increased to a one in four risk

15 Bauchner H, Vinci R. What have we learnt from the Alder Hey affair? BMJ 2001;322:309-10.

(Accepted 7 November 2003)

doi 10.1136/bmj.37939.570104.EE

National Perinatal Epidemiology Unit, Institute of Health Sciences, Headington, Oxford OX3 7LF

P A Boyd senior clinical research fellow

Dipartimento di Ginecologia, Perinatologia e Riproduzione umana, Facolta di

Medicina e Chirurgia, Universita degli Studi di Firenze, Viale Morgagni, 85,

Florence

F Tondi medical student

Division of Public Health and Primary Care, Institute of Health Sciences,

University of Oxford, Headington, Oxford OX3 7LF

N R Hicks homorary senior clinical lecturer

Prenatal Diagnosis Unit, Women's Centre, Oxford Radcliffe NHS Trust, Oxford OX3 9DU

P F Chamberlain consultant obstetrician

Correspondence to: P Boyd Patricia.boyd@orh.nhs.uk 